\title{
Trauma Spillover in Social Networks
}

\section{Sadish}

October 11, 2021

Internet searches indicative of mental disorders increased in US counties when COVID-19 casualties rose in other places - inside and outside US borderswith which they had strong ties over social media. Casualties within counties or in their vicinity, the stringency of COVID-19 response policies, and the seasonality of affective disorders do not account for the association; nor do characteristics fixed to counties or time. Social ties as defined by migration confirm the association. The results merit attention because internet search behavior predicts emergency department visits.

Keywords: Mental Health, Social Networks, COVID-19

•[sadishdhakal@gmail.com] 


\section{Acknowledgement}

I thank Jimmy Narang and Pedro CL Souza for comments.

\section{Conflict of Interest}

I have no source of funding or conflict of interest to declare concerning this study.

\section{Ethical Review}

This study did not undergo ethical review.

\section{Data Availability}

All data used in this study are publicly available. 
The internet lets us vicariously experience distant events in real time. We can commiserate or celebrate with friends far away through social media, evidently to such an extent that we feel what they feel. One global experiment, though its ethics came under question, showed that varying social media users' exposure to expressions of positive or negative emotions from their acquaintances led them to imbibe and express similar emotions [1]. The study lent credence to the idea that emotions can spread like contagions in social networks [2]. Previous evidence in support of the view was largely correlational [3]. Some studies have gone further and proposed that affective disorders like depression [4] and loneliness [5] can transmit through social networks.

If real, the phenomenon could have substantial public health consequences, particularly in times of crisis. Terrorist attacks [6, 7], natural disasters [8], and pandemics $[9,10]$ are known to entail widespread mental illnesses where they occur. Could the trauma of such crises flow through social networks that transcend physical and political borders? One study showed that, immediately following the September 11, 2001 terrorist attack in the US, trauma-related mental disorders surged in Denmark [11]. Another found that refugees used more antidepressants and anxiolytic drugs when terrorism in their home countries intensified [12]. Here, I further investigate the question.

My inquiry is made possible by new, publicly available datasets with high spatiotemporal resolution.

Internet Searches Indicative of Symptoms: In 2020, Google began releasing weekly data on the prevalence of internet searches related to over 400 health conditions, including mental disorders, in each US county. These data are valuable because they represent private health information seeking behavior. In 2020, queries on Google's platform accounted for $88 \%$ of internet searches in the US [13]. Researchers have shown that data from internet search engines and social media can be used for public health surveillance of infectious diseases $[14,15]$, though others [16] have also cautioned us not to take the construct validity of internet search behavior for granted.

Figure 1 confirms the data's validity. It shows that internet searches indicative of certain mental disorders correlated substantially with the number of under-18 individuals visiting emergency departments with disaster-related mental health 
problems [17], even through the disruptive onset of the pandemic. (See Section 1 for further discussion on convergent and discriminant validity.)

Figure 1: Internet Searches Indicative of Mental Disorders and Emergency Department Visits with Disaster-Related Mental Health Problems among Individuals Under 18 Over Time

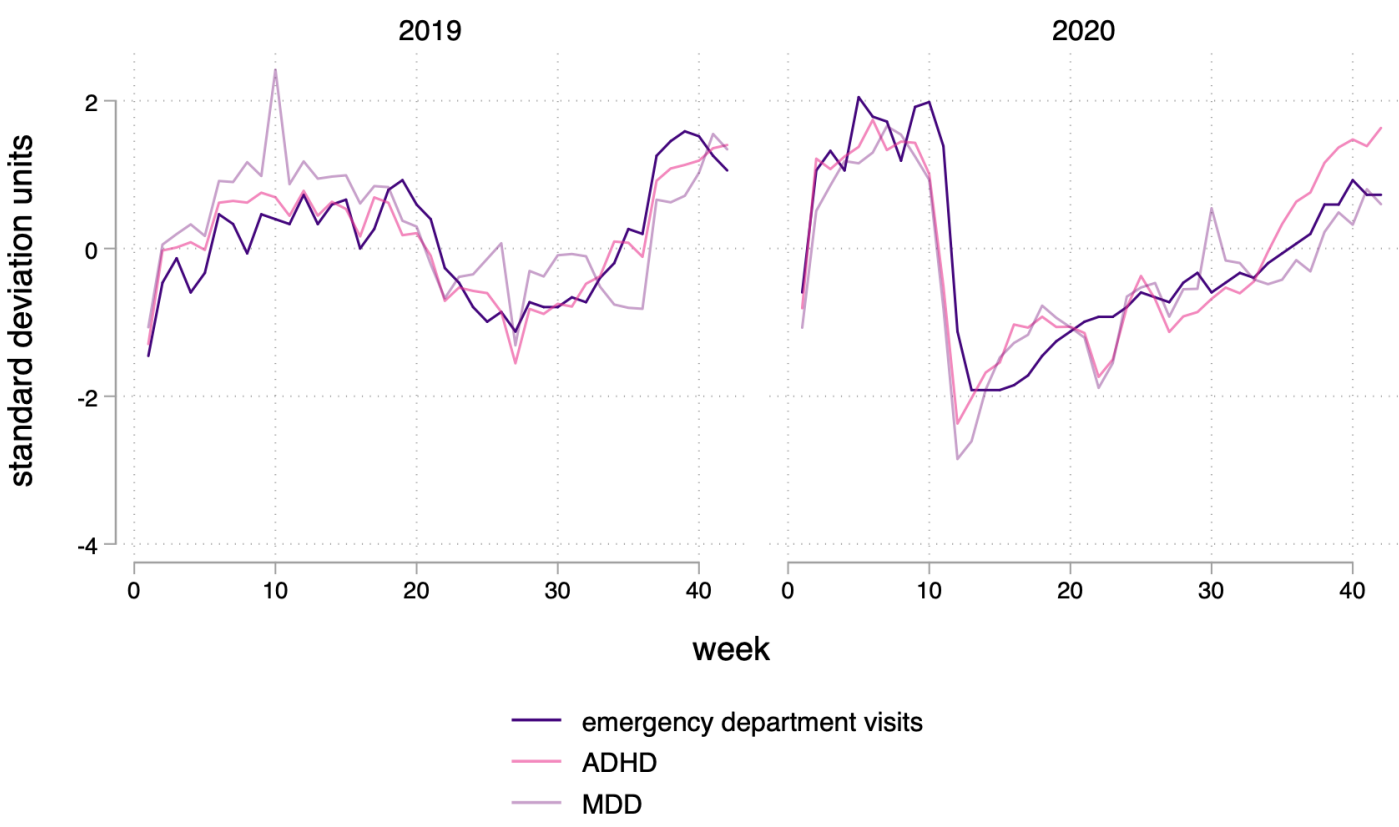

Weekly aggregated data on emergency department visits from 47 US states were available only for the first 42 weeks of 2019 and 2020 .

Social Network: In 2020, Facebook updated the 2018 release of its social connectedness index. The index is a measure of the density of Facebook connections between pairs of US counties, and between US counties and countries worldwide. The data represent the social networks of $36 \%$ of the world's population - 2.8 billion people - who use Facebook every month [18]. Researchers have established the index's validity in different contexts. In the US and Europe, the index correlates with the similarity of two locations vis a vis 
history, language, age, religion, political affiliations, education, income, and population movement [19-21]. Globally, it correlates with the volume of trade between countries [22]. It has been shown to moderate the spread of COVID-19 [23] and compliance with lockdown policies [24].

Severity of the COVID-19 Pandemic: In 2020, the Open Data project began compiling, among other data, the number of COVID-19 cases, COVID19 deaths, and the stringency of governments' policy response to the pandemic globally. While COVID-19 cases and deaths measure the pandemic's severity imperfectly, the data are well documented. Alternatives such as hospitalizations and excess deaths are as yet sparse, unavailable at sufficiently high geographic and temporal resolutions, and come with their own measurement errors.

(See Section 1 for detailed descriptions of the data and data processing procedures.)

Equipped with these data, imagine that counties and countries are nodes linked together in a social network. The natural log of the social connectedness index [19, 20, 22, 24] - a link's weight - quantifies the locations' influence on each other. A county's exposure to the pandemic in another location is simply the latter's pandemic severity multiplied by its influence. A county's exposure to the pandemic in its entire network, then, is the sum of its exposure to each of the other locations. Consequently, if the severity is constant for all locations, those with stronger social ties experience higher exposure. I distinguish between local exposure - counties' exposure to other counties, and global exposure- counties' exposure to countries worldwide.

I model internet search behavior indicative of mental disorders as a linear function of counties' exposure to COVID-19 cases or deaths. I control for deaths or cases within the counties' own borders, spatial and temporal lags of search behavior, the stringency of US states' COVID-19 control policies, hours of daylight, unobserved effects fixed to counties, and unobserved effects fixed to time.

The model draws from the literature on social networks, peer effects, and neighborhood effects [25-28]. It is the social networks equivalent of the SLX approach in spatial econometrics, and is suited for my purpose based on prior theoretical exposition $[29,30]$. (See section 1 for precise descriptions of the variables and the model.)

I estimate the model using least squares regression. Taking guidance from recent 
research [31], I calculate heteroskedasticity and autocorrelation consistent standard errors using previously developed procedures $[32]^{1}$. Figure 2 shows the estimated coefficients and standard errors.

The figure provides strong evidence that internet searches indicative of most mental disorders rose with global exposure to the pandemic, though the estimates for alcoholism and sleep related disorders are less consistent. Search behavior related to sex and sleep rose with local exposure to the pandemic but those related to other mental disorders did not.

Figure 2: Association Between Internet Searches Indicative of Mental Disorders and Exposure to the COVID-19 Pandemic
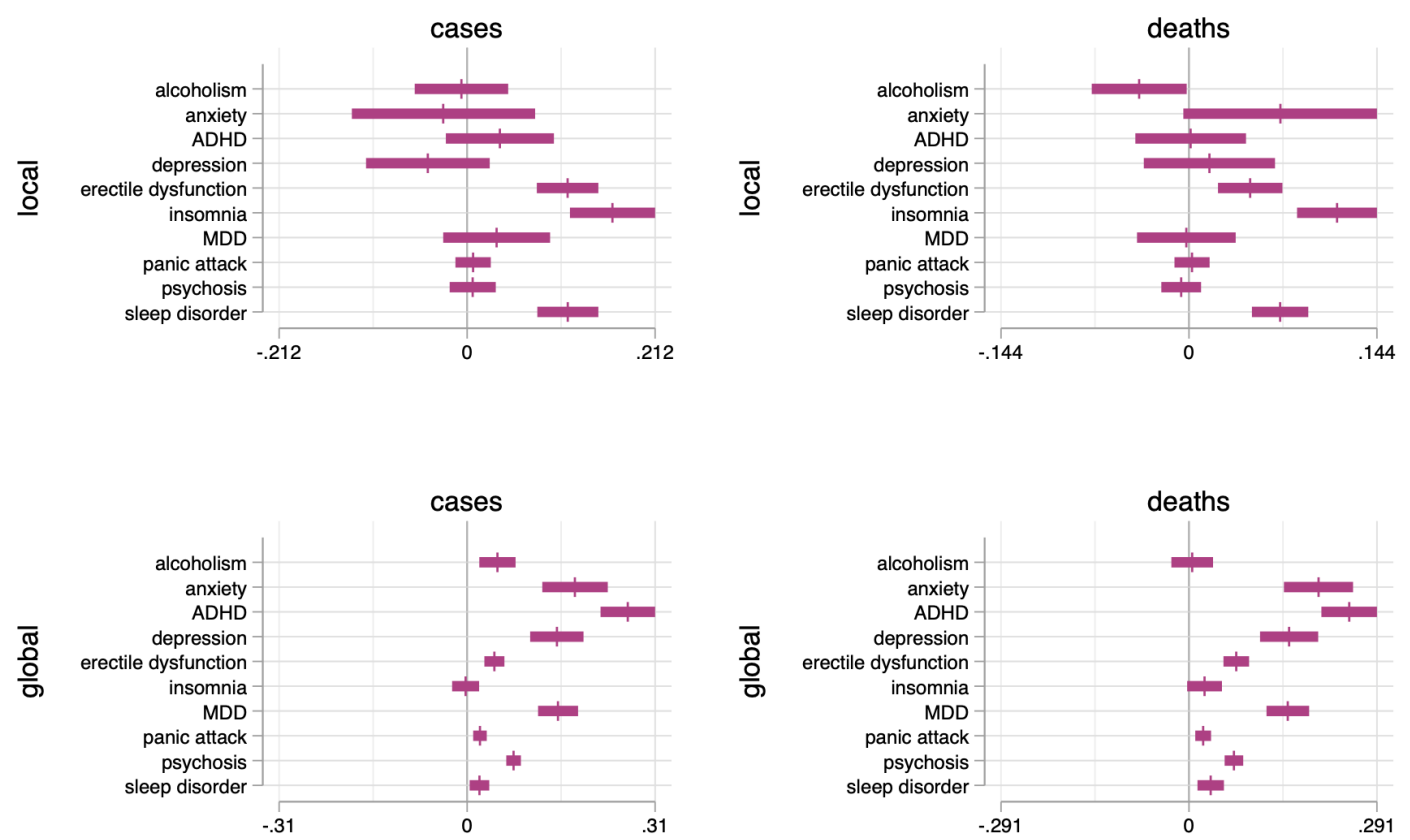

The figure shows coefficient estimates (vertical markers) and 95 percent confidence intervals (horizontal bands) using heteroskedasticity and autocorrelation consistent standard errors. The x-axis

\footnotetext{
${ }^{1}$ I allow for spatial correlations within 600 kilometers of counties and temporal correlations with up to 12 preceding weeks.
} 
is in units of standard deviation and its scale is unique to each plot.

I test for the results' sensitivity to various assumptions (See Section 2). Controlling for unemployment rate and exposure to national and international COVID-19 response policies do not challenge the results. Estimating the model using median regression corroborates the findings. Confidence intervals are wider when clustering standard errors by county and date, but not enough to cast doubt on the conclusions. Finally, I estimate the model using an alternative measure of social network weights - the scale of migration between locations - derived from survey data. The estimates, shown in Figure 3, validate the results with a few exceptions pertaining to local exposure.

Figure 3: Association Between Internet Searches Indicative of Mental Disorders and Exposure to the COVID-19 Pandemic (Migration Network)
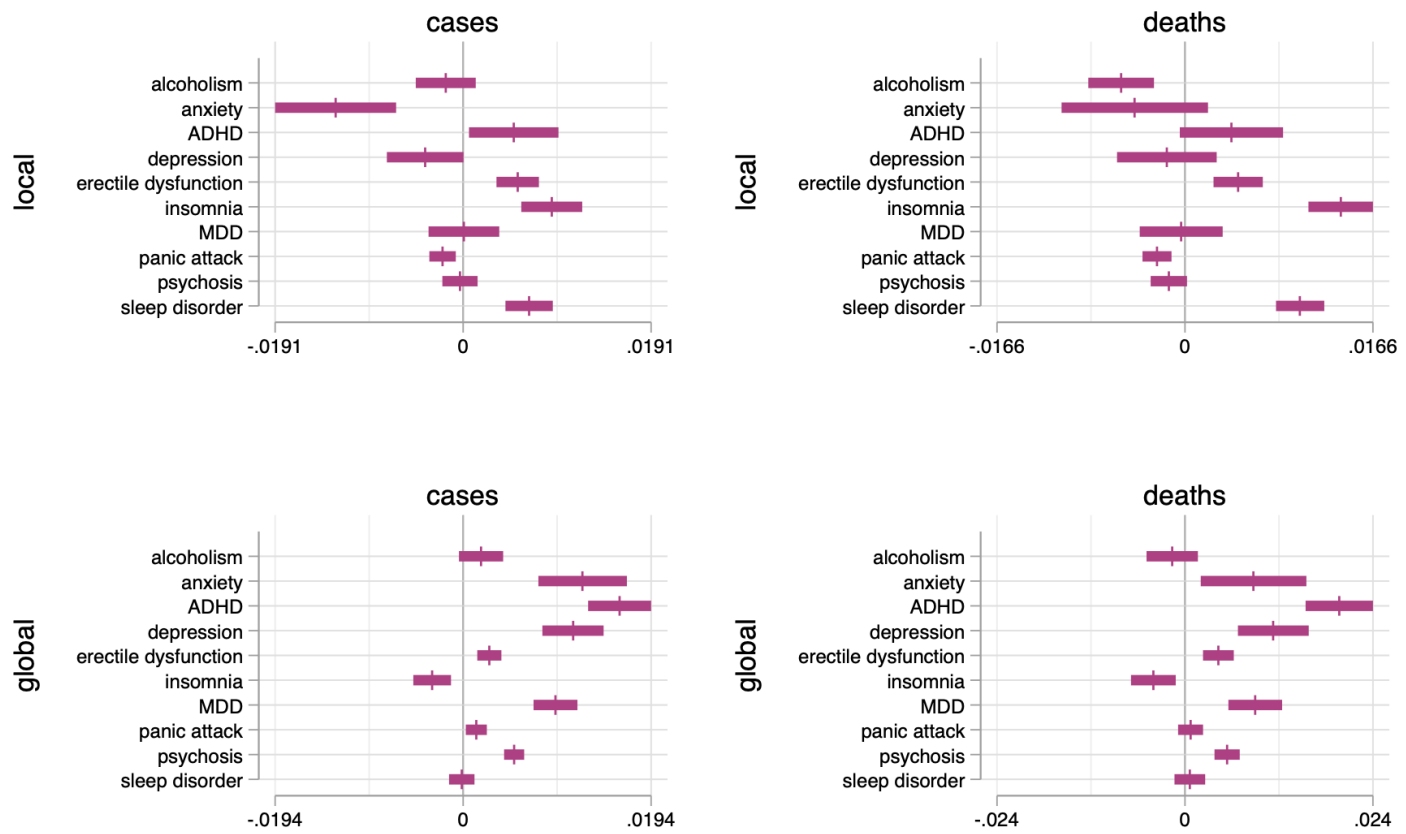

The figure shows coefficient estimates (vertical markers) and 95 percent confidence intervals (horizontal bands) using heteroskedasticity and autocorrelation consistent standard errors. The $\mathrm{x}$-axis 
is in units of standard deviation and its scale is unique to each plot.

I uncover an association between internet search behavior indicative of mental disorders and exposure to the COVID-19 pandemic via social networks. Since such search behavior correlate with the number of mental health related emergency department visits, I take search behavior to be a valid measure of the prevalence of mental disorders in the population. My findings, then, are consistent with the proposition that trauma spills over in social networks.

A variable omitted from my model, if it correlates with both mental health and exposure, could account for the association. The question is not whether such a variable exists, but what it is. Here, my analysis confronts limitations. As a county's exposure rises, its residents could have higher chances of knowing persons affected by COVID-19 or they could encounter more media coverage of the pandemic; either may affect mental health $[1,7,8]$.

\section{Data and Methods}

\subsection{Data}

I combine data from several sources. All analyses, with the exception of that in Figure 1, are based on data representing about 3,000 US counties and 200 countries during 70 weeks from mid-March 2020 onward.

\section{Internet Searches Indicative of Symptoms}

In 2020, Google began releasing data on internet searches related to over 400 health symptoms for six countries at varying geographic resolutions [33]. The data span over three years and are available at day or week levels of aggregation. To calculate the index, Google divided the total number of searches ascribed to a given symptom in a given geographic unit in a given time period by the total number of searches in that geographic unit in that time period. In the initial release, the ratio was scaled so that the maximum for each geographic unit was 100. Later releases may have values higher than 100 if the ratio exceeded its initial maximum. Searches by a single user on a given day were counted only once per symptom for up to three symptoms. Google added artificial noise to the data to preserve the privacy of individuals and did not report data when there were too few searches for a particular symptom in a given location at a given time. This censoring means data are not missing at random. Missing data represent the lowest search volumes. 
I use the index from US counties aggregated weekly, except in Figure 1 where I aggregate weekly data at the state level. Given the high geographic resolution of US counties, weekly aggregation makes it more feasible to preserve privacy with minimal artificial noise and censoring [33]. For this reason, weekly data have fewer missing values.

With over 400 symptoms to choose from, a researcher may retroactively justify associations between an explanatory variable and a set of symptoms. A researcher looking for associations between a random variable and 400 other random variables will find 20 statistically significant associations at a $5 \%$ level of significance. Exacerbating the problem, a wide array of symptoms accompany mental illnesses. Headaches, dizziness, nausea, tinnitus, perspiration, tremors, as well as gastrointestinal problems such as constipation, stomach ache, and diarrhea are all associated with stress and mental disorders $[34,35]$.

I first discard all symptoms with data missing for more than $33 \%$ of observations, leaving me with a fifth of them. From the remaining, I select mental disorders defined as such by the American Psychiatric Association in the Diagnostic and Statistical Manual of Mental Disorders [34]. These disorders are: alcoholism, attention deficit hyperactivity disorder (ADHD), erectile dysfunction, major depressive disorder (MDD), panic attack, and insomnia. The earlier procedure removes suicidal ideation and self-harm because they have too many missing values. I include anxiety, depression, psychosis, and sleep disorder which map to a broader range of disorders in the manual. Though the World Health Organization's Classification of Mental and Behavioral Disorders uses a slightly different taxonomy, the selected symptoms align with its definitions of mental disorders as well [35]. I set missing values to the lowest non-missing value in the location and standardize each symptom to have mean zero and standard deviation one.

Figure 4 shows high volume of searches related to COVID-19 during the beginning of the pandemic. The pattern is absent for mental disorders. Search behavior was similar for anxiety and depression, as well as for ADHD and MDD, particularly at the end of 2020. 
Figure 4: Trends in Internet Searches Indicative of Symptoms
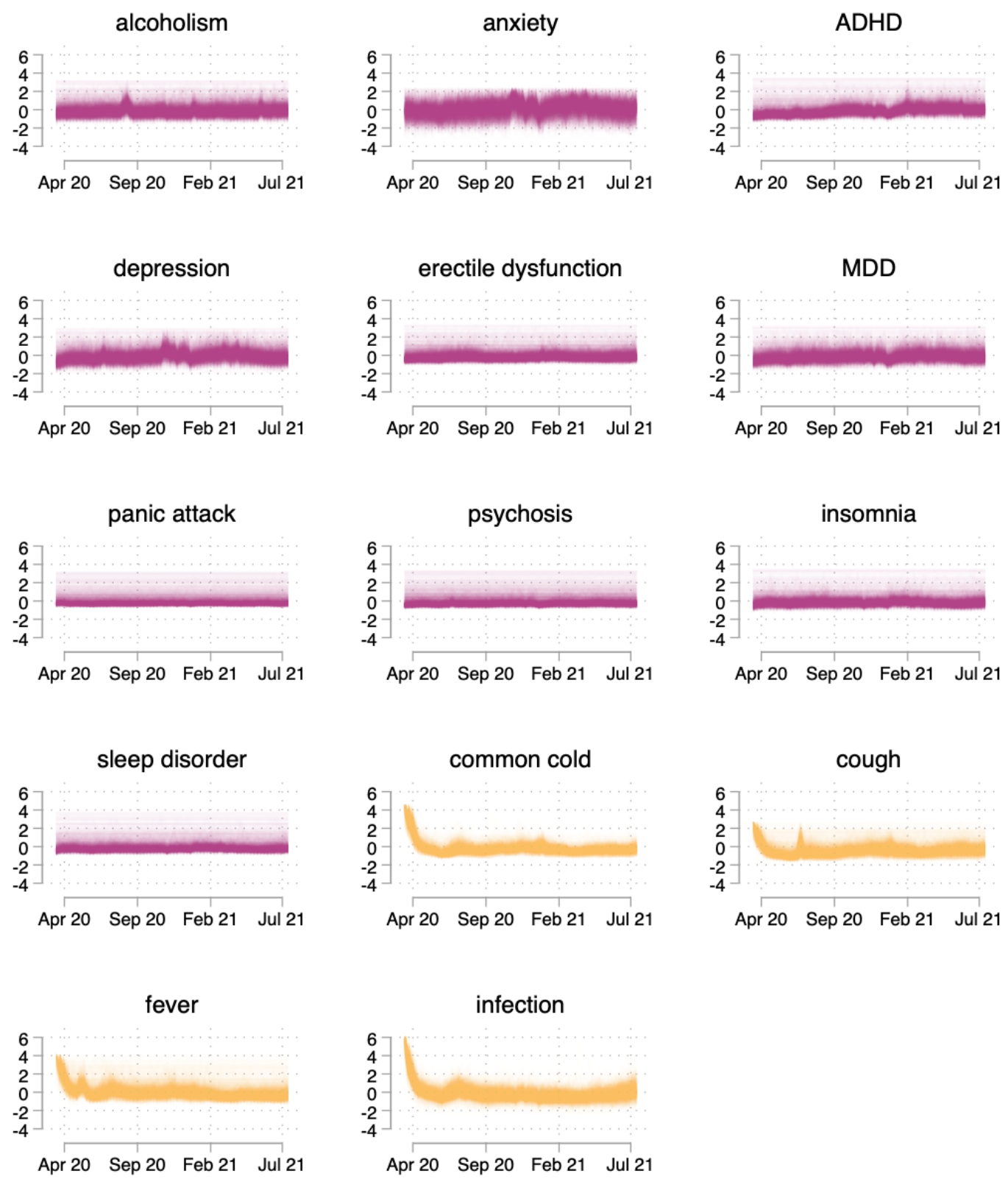
Y-axis is in units of standard deviation. X-axis is in unit of weeks. Visualization excludes values above the $98^{\text {th }}$ percentile. Colors distinguish between mental disorders and COVID-19 symptoms.

Figure 5 shows correlations between search behavior related to mental disorders and, for comparison, COVID-19 symptoms: common cold, cough, fever, and infection. Search behavior related to COVID-19 symptoms are strongly correlated with each other. Mental disorders are also correlated with each other, albeit to a lesser degree. Correlations between mental disorders and COVID-19 symptoms are minimal.

Principal Component Analysis on these symptoms shows that the first two principal components explain $67 \%$ of the variation, and the first three explain $76 \%$. Loadings on the first two principal components in Figure 5 clearly distinguish between searches related to mental disorders and COVID-19 symptoms. The analysis confirms that the data have convergent and discriminant validity. 
Figure 5: Correlations Between Symptoms and Principal Component Loadings
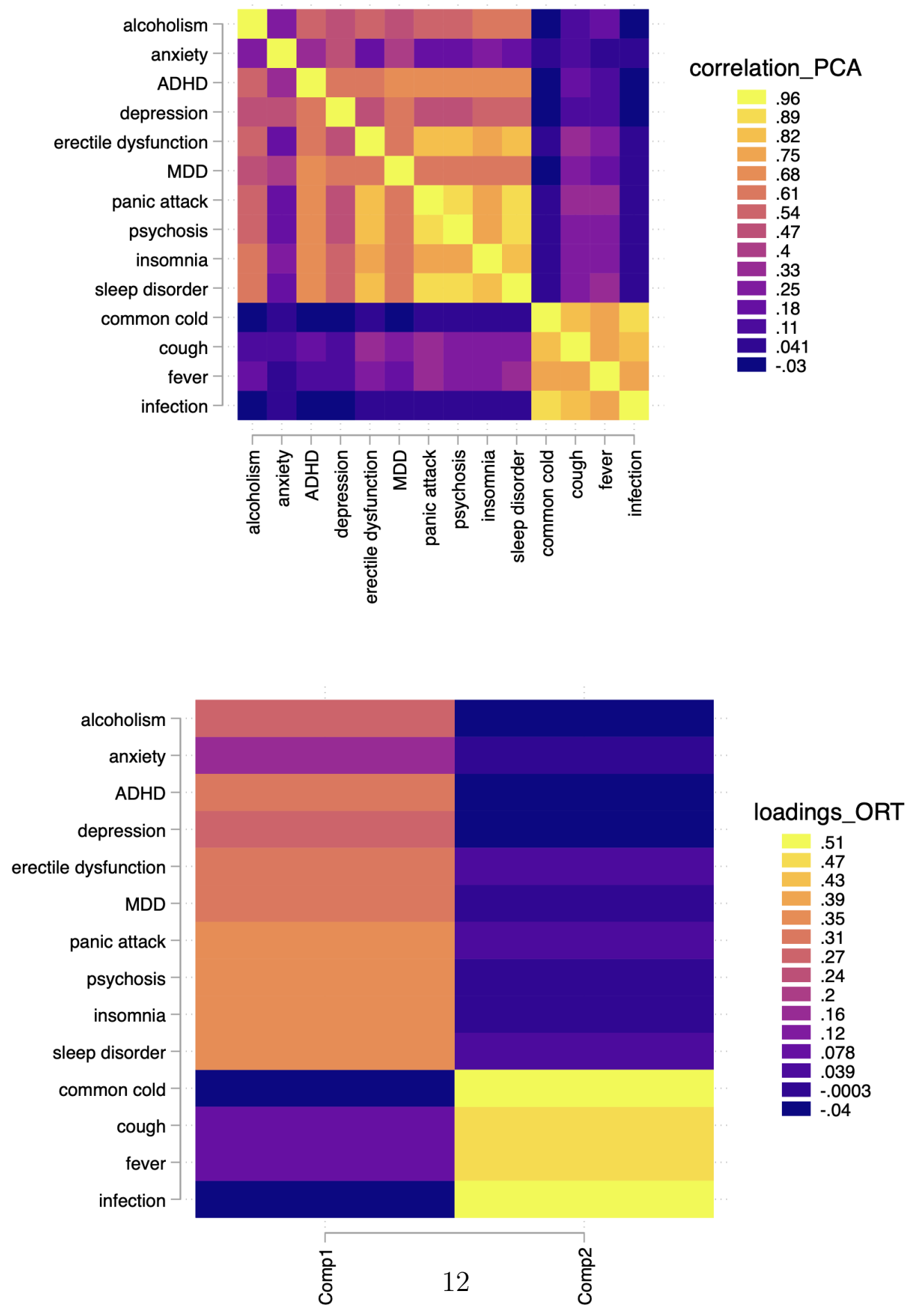
The figure shows correlations between symptoms and loadings on the first two principal components.

\section{Social Network}

Facebook released the first version of the Social Connectedness Index in 2018 [19]. The index is the ratio of the number of Facebook connections between residents of two locations to the number of possible connections between Facebook users in these locations, scaled to have a minimum value of 1 and maximum value of 1 billion [36]. It can be interpreted as the probability of there existing a social connection between individuals residing in two locations, or as the density of social connections between the locations.

In late 2020, Facebook released datasets in varied geographic resolutions [36]. I use two datasets: the first defines the index for pairs of US counties, and the second for US counties paired with countries. Each dataset uses a unique scaling, making the indices incomparable across them. Following various applications of the social connectedness index by its original authors [19, 20, 22] and others [24], I take its natural log as a measure of social network weights.

Figure 6 shows that the strongest links in the local social network exist between the closest counties, especially in central US. State borders and geographical features act as barriers. Figure 7 shows counties' links with Canada and Mexico. Counties closest to a country's border are most strongly linked with it. 
Figure 6: Local Social Network Links

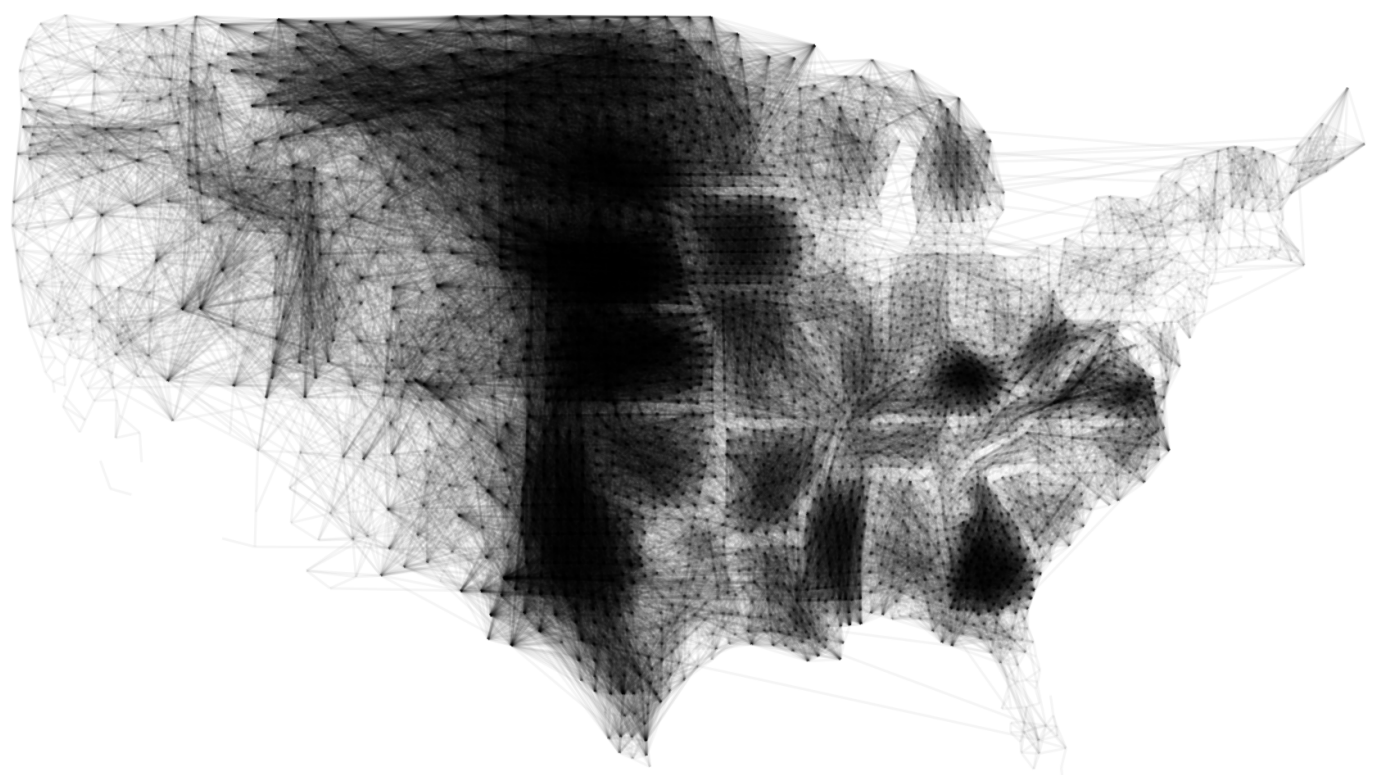

The figure shows social network links between counties in the contiguous US with the highest $2 \%$ of weights. 
Figure 7: Global Social Network Links

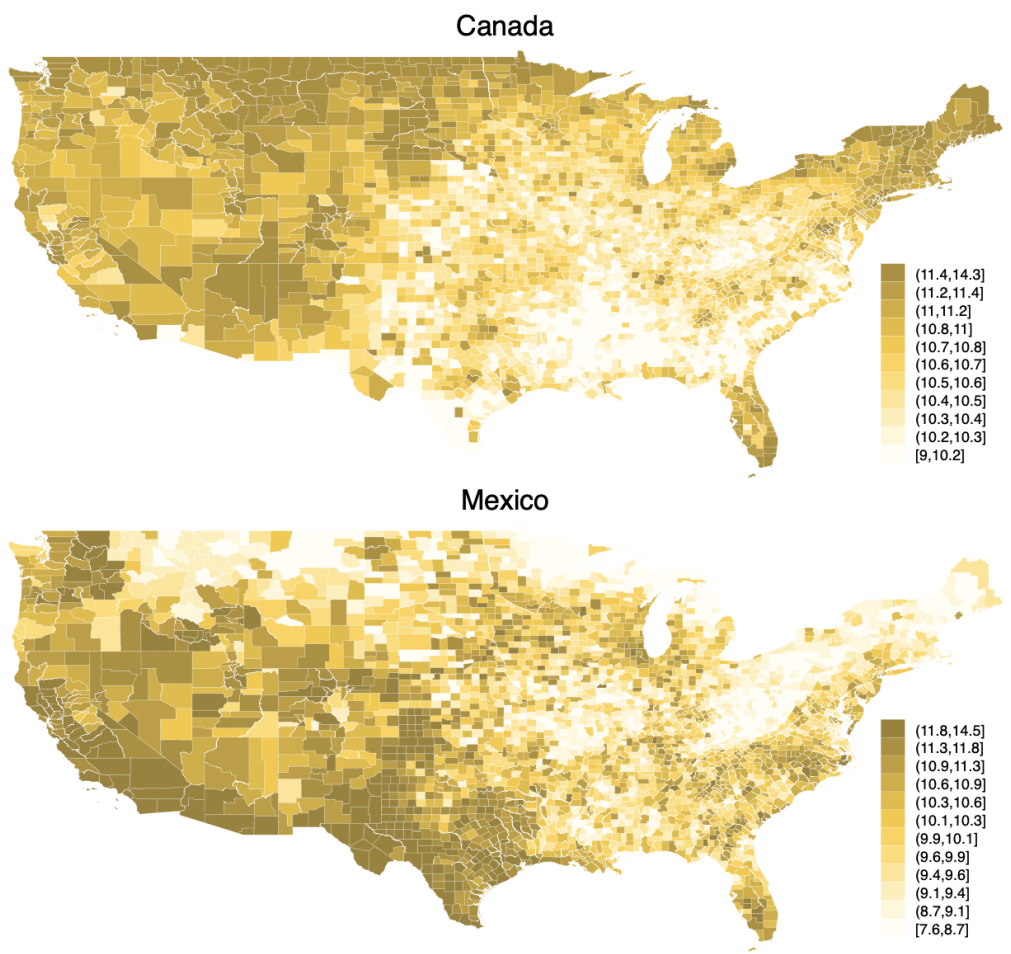

The figure shows the weights of counties' social network links with Canada and Mexico.

\section{Severity of the COVID-19 Pandemic}

The COVID-19 Open Data project [37] has curated data on COVID-19 cases and deaths from inter-governmental organizations [38, 39], government agencies, and other sources $[40,41]^{2}$. I use new cases and deaths per day as measures of pandemic severity. Figure 8 shows that the pandemic's toll varied widely in both magnitude and timing.

\footnotetext{
${ }^{2}$ full list of sources of casualty data [retrieved 2021-03-03]:

https://github.com/GoogleCloudPlatform/covid-19-open-data/blob/main/docs/tableepidemiology.md
} 
Figure 8: Trends in COVID-19 Casualties
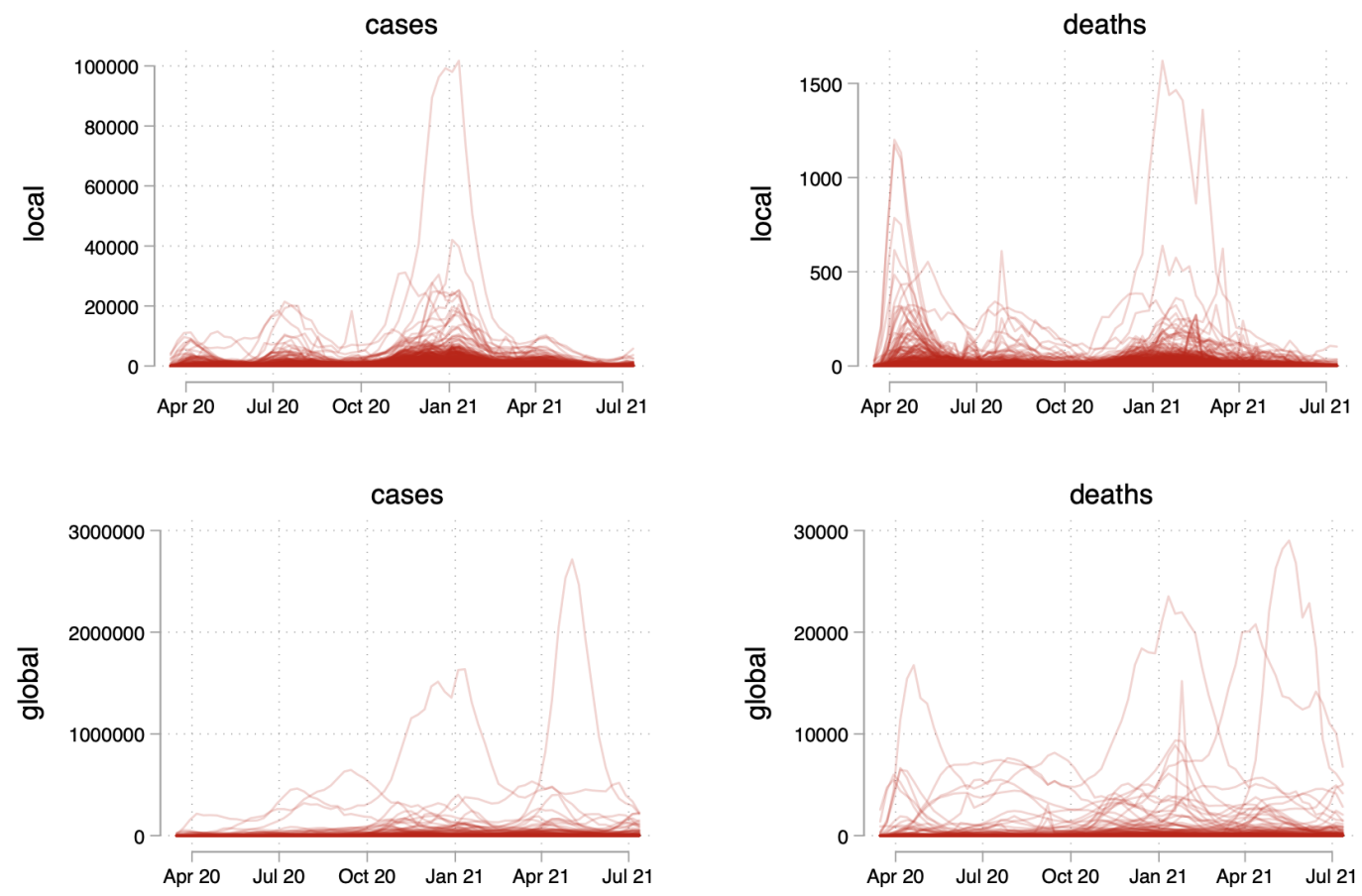

Y-axis shows counts per day and its scale is unique to each plot. $\mathrm{X}$-axis is in units of weeks.

\section{Other Data}

Policy Response to COVID-19: Researchers from Oxford University have developed an index that measures the stringency of the policy response to the COVID-19 pandemic $[37,42]$. I use it as a control variable.

Emergency Department Visits: The US Centers for Disease Control and Prevention's National Syndromic Surveillance Program has data on emergency department visits for 47 states in the US. The published data [17] provide the weekly total number of emergency department visits made by individuals under 18 years of age with mental health conditions that are likely to follow disaster events - stress, anxiety, acute posttraumatic stress disorder, and panic for instance. Data are available for the first 42 weeks of 2019 and for the same period in 2020. I study the correlation 
between emergency department visits and internet search behavior.

Migration: I use US Census Bureau's data on gross migration between counties [43] and data from IPUMS on the number of county residents born in each foreign country [44]. I take the natural log of these estimates as measures of the migration network weights.

Geographical Data: I use cartographic boundaries for US counties from the US Census Bureau [45]. I use geographic coordinates [37] to generate spatial lags and to calculate hours of daylight. I use the International Standardization Organization's 2-digit country codes and Federal Information Processing Standard codes to combine data from different sources.

\subsection{Model}

Let $i$ be a US county, the unit under study. At time $t$, county $i$ has a volume of internet search behavior indicative of health symptoms $s_{i t}$ and experiences the pandemic with severity $z_{i t}$ within its own borders. Through the social network, county $i$ is also exposed to $z_{j t}$ and $z_{k t}$ - the severity of the pandemic at county $j$ and country $k$ respectively.

Suppose $w_{i j}$ is the weight of the social network link between $j$ and $i, w_{i k}$ is that between $k$ and $i$, and suppose that $d_{i j}$ is the distance between $j$ and $i$. Let, $\boldsymbol{x}$ be a $1 \times m$ vector of $m$ variables. I estimate the following model: 


$$
\begin{aligned}
s_{i t}= & & & \text { search behavior } \\
& + & & \text { constant } \\
& +\psi \sum_{j \neq i} w_{i j} z_{j t} & & \text { local exposure } \\
& +\phi \sum_{k} w_{i k} z_{k t} & & \text { global exposure } \\
& & & \\
& +\sum_{p=1}^{2} \gamma_{p} s_{i(t-p)} & & \text { temporal lags } \\
& +\delta \sum_{j \neq i} \frac{s_{j t}}{1+d_{i j}} & & \text { spatial lag } \\
& +\boldsymbol{x} \boldsymbol{\beta} & & \text { controls } \\
+ & & & \text { unobserved error fixed to county } i \\
& +\iota_{t} & & \text { unobserved error fixed to time } t \\
& +\epsilon_{i t} & & \text { residual unobserved error }
\end{aligned}
$$

Here, $\alpha, \theta, \psi, \phi, \delta$, and $\gamma_{p}$ are parameters to be estimated; as are the elements of the $m \times 1$ vector $\boldsymbol{\beta}$.

My objective is to estimate the total effect [46] of $\sum_{j \neq i} w_{i j} z_{j t}$ and $\sum_{k} w_{i k} z_{k t}$ on $s_{i t}$, not to separate the direct effects from the indirect effects mediated by $\sum_{j} w_{i j} s_{j t}$, which I observe, and $\sum_{k} w_{i k} s_{k t}$, which I do not. The total effects are identified when I omit $\sum_{j} w_{i j} s_{j t}$ and $\sum_{k} w_{i k} s_{k t}$ from the model [46]. Introducing the terms in the model is akin to the fallacy of conditioning on post-treatment variables [47] or on intermediate outcomes [48].

\section{Sensitivity to Assumptions}

I carry out tests to check whether the results are sensitive to various assumptions.

Additional Control Variables: Since governments modulated COVID-19 response policies based on the severity of the pandemic, exposure to such policies could be correlated with exposure to the pandemic's severity. I control for global and local 
exposure to COVID-19 response policies through social and spatial networks. In the same specification, I also control for monthly county-level unemployment rates to account for economic shocks. These variables do not account for the association between exposure to the pandemic and internet searches indicative of mental disorders. (see Figure 9)

Figure 9: Association Between Internet Searches Indicative of Symptoms and Exposure to the COVID-19 Pandemic (Additional Control Variables)
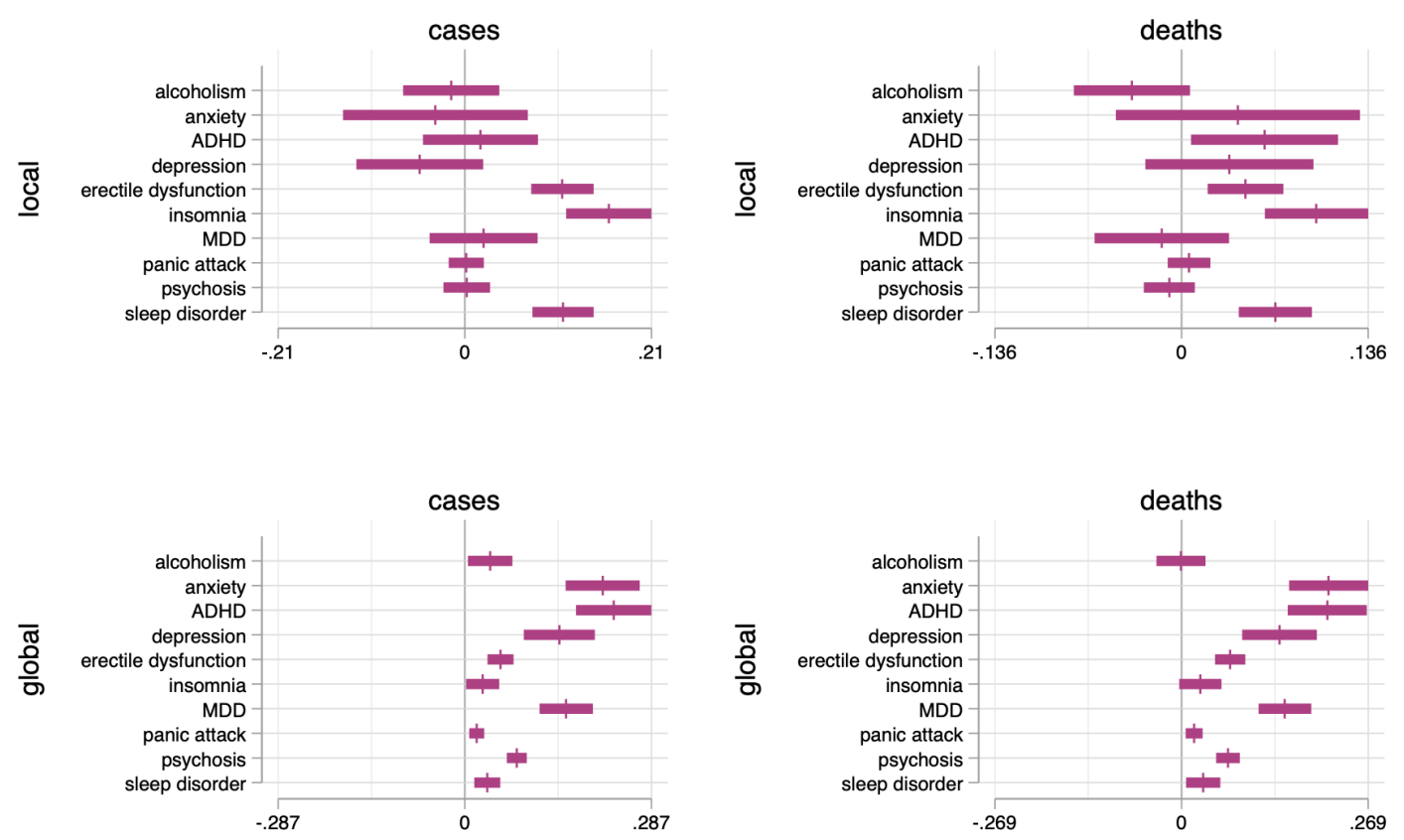

The figure shows coefficient estimates (vertical markers) and 95 percent confidence intervals (horizontal bands) using heteroskedasticity and autocorrelation consistent standard errors. The x-axis is in units of standard deviation and its scale is unique to each plot. Additional control variables are local and global exposure to COVID-19 response policies, as well as monthly county-level unemployment rates.

Median Regression: Internet search indices are somewhat positively skewed because the lowest values are censored to preserve privacy. To limit the influence of the 
largest values, I estimate the model using median regression. These specifications corroborate the results. (see Figure 10)

Figure 10: Association Between Internet Searches Indicative of Symptoms and Exposure to the COVID-19 Pandemic (Median Regression)
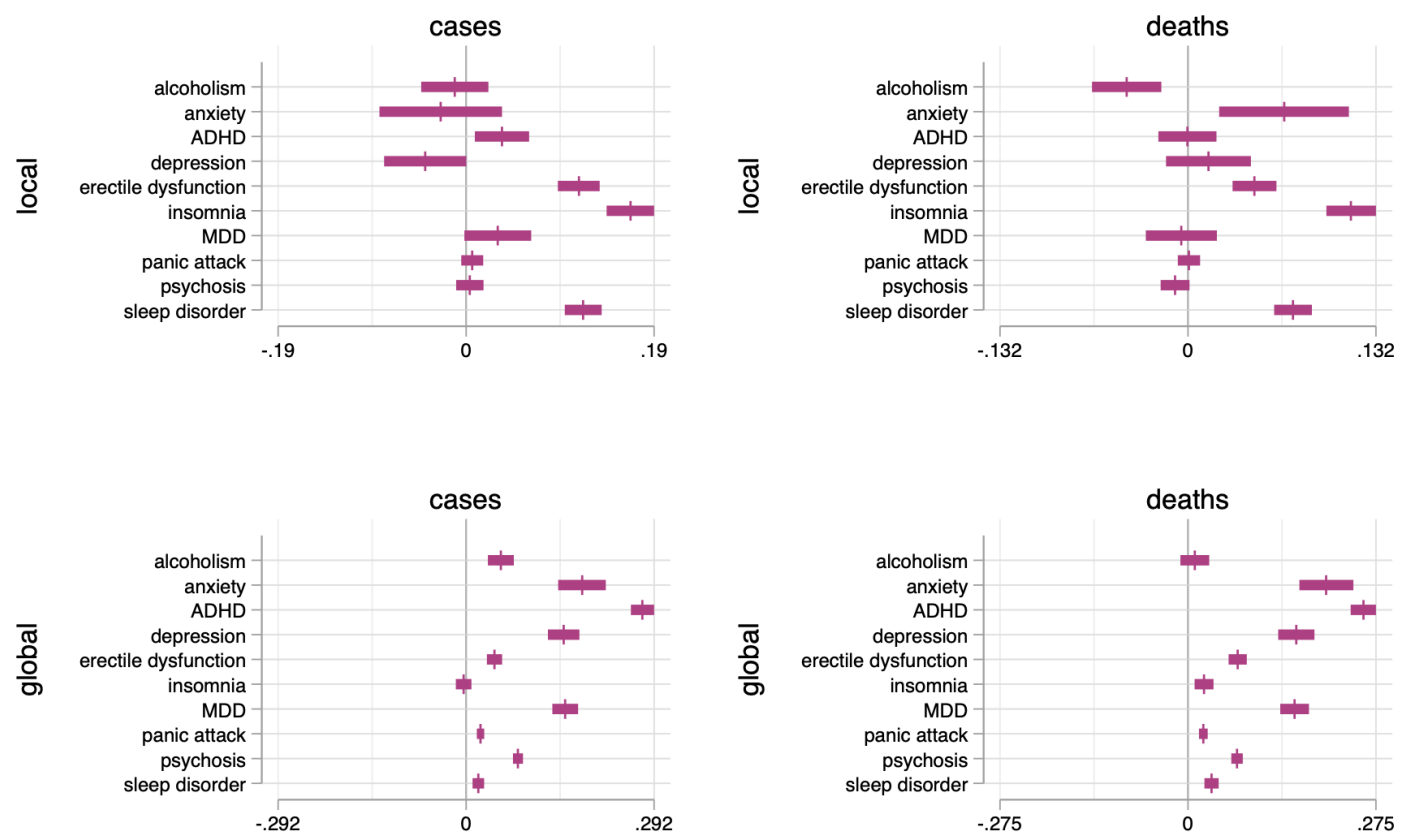

The figure shows coefficient estimates (vertical markers) and 95 percent confidence intervals (horizontal bands). The x-axis is in units of standard deviation and its scale is unique to each plot.

Standard Errors: I estimate the main specification by allowing standard errors to cluster by county and date. Allowing for clustering produces wider confidence intervals, though not enough to cast doubt on the results. (see Figure 11) 
Figure 11: Association Between Internet Searches Indicative of Symptoms and Exposure to the COVID-19 Pandemic (Clustered Standard Errors)
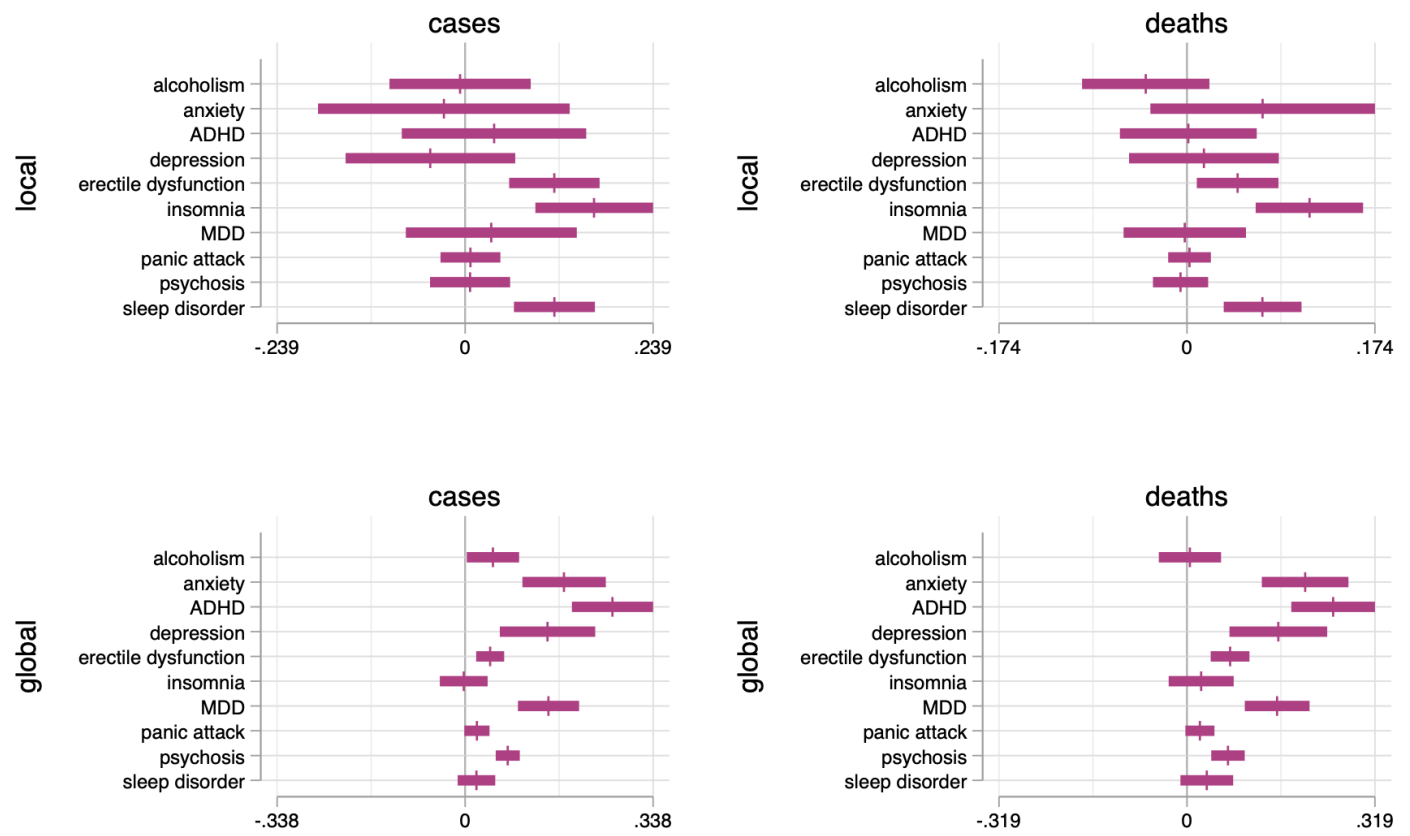

The figure shows coefficient estimates (vertical markers) and 95 percent confidence intervals (horizontal bands) using date and county clustered standard errors. The x-axis is in units of standard deviation and its scale is unique to each plot. 


\section{References}

1. Kramer, A. D. I., Guillory, J. E. \& T. Hancock, J. Experimental Evidence of Massive-Scale Emotional Contagion Through Social Networks. Proceedings of the National Academy of Sciences 111, 8788-8790 (2014).

2. Hatfield, E., Cacioppo, J. T. \& Rapson, R. L. Emotional Contagion. Current Directions in Psychological Science 2, 96-99 (1993).

3. Fowler, J. H. \& Christakis, N. A. Dynamic Spread of Happiness in a Large Social Network: Longitudinal Analysis Over 20 Years in the Framingham Heart Study. BMJ 337, 1-9 (2008).

4. Rosenquist, J., Fowler, J. \& Christakis, N. Social Network Determinants of Depression. Molecular Psychiatry 16, 273-281 (2011).

5. Cacioppo, J. T., Fowler, J. H. \& Christakis, N. A. Alone in the Crowd: The Structure and Spread of Loneliness in a Large Social Network. Journal of Personality and Social Psychology 97, 977-991 (2009).

6. Holman, E. A., Garfin, D. R. \& Silver, R. C. Media's Role in Broadcasting Acute Stress Following the Boston Marathon Bombings. Proceedings of the National Academy of Sciences 111, 93-98 (2013).

7. Thompson, R. R., Jones, N. M., Holman, E. A. \& Silver, R. C. Media Exposure to Mass Violence Events Can Fuel a Cycle of Distress. Science Advances 5, 1-6 (2019).

8. Thompson, R. R., Holman, E. A. \& Silver, R. C. Media Coverage, Forecasted Posttraumatic Stress Symptoms, and Psychological Responses Before and After an Approaching Hurricane. JAMA Network Open 2, 1-12 (2019).

9. Holman, E. A., Thompson, R. R., Garfin, D. R. \& Silver, R. C. The Unfolding COVID-19 Pandemic: A Probability-Based, Nationally Representative Study of Mental Health in the United States. Science Advances 6, 1-7 (2020).

10. Thompson, R. R., Garfin, D. R., Holman, E. A. \& Silver, R. C. Distress, Worry, and Functioning Following a Global Health Crisis: A National Study of Americans' Responses to Ebola. Clinical Psychological Science 5, 513-521 (2017).

11. Hansen, B. T., Ostergaard, S. D., Sonderskov, K. M. \& Dinesen, P. T. Increased Incidence Rate of Trauma- and Stressor-Related Disorders in Denmark After the September 11, 2001, Terrorist Attacks in the United States. American Journal of Epidemiology 184, 494-500 (2016).

12. Sonderskov, K. M., Dinesen, P. T., Hansen, B. T., Ostergaard, S. D. \& Danckert, B. Terrorism in The Country of Origin Is Linked to Deterioration in The Mental Health of Refugees. Nature Human Behavior, 1-7 (2021). 
13. Statcounter. Search Engine Market Share United States Of America https: //gs.statcounter.com/search-engine-market-share/ (2021).

14. Ginsberg, J. et al. Detecting Influenza Epidemics Using Search Engine Query Data. Nature 457, 1012-1015 (2009).

15. Aiello, A. E., Renson, A. \& Zivich, P. N. Social Media- and Internet-Based Disease Surveillance for Public Health. Annual Review of Public Health 41, 101-118 (2020).

16. Lazer, D., Kennedy, R., King, G. \& Vespignani, A. The Parable of Google Flu: Traps in Big Data Analysis. Science 343, 1203-1205 (2014).

17. Leeb, R. T. et al. Mental Health-Related Emergency Department Visits Among Children Aged <18 Years During the COVID-19 Pandemic — United States, January 1-October 17, 2020. Morbidity and Mortality Weekly Report 69, 16751680 (2020).

18. Facebook. Facebook Reports Fourth Quarter and Full Year 2020 Results https:

/ / investor . fb . com / investor-news / press - release-details / 2021 / Facebook - Reports - Fourth - Quarter - and - Full - Year - 2020 - Results / default.aspx (2021).

19. Bailey, M., Cao, R., Kuchler, T., Stroebel, J. \& Wong, A. Social Connectedness: Measurement, Determinants, and Effects. Journal of Economic Perspectives 32, 259-280 (2018).

20. Bailey, M. et al. The Determinants of Social Connectedness in Europe. Social Informatics, 1-14. https://link.springer.com/chapter/10.1007/978-3030-60975-7_1 (2021) (2020).

21. Li, Z. et al. Measuring Global Multi-Scale Place Connectivity Using Geotagged Social Media Data. Scientific Reports 11, 1-19 (2021).

22. Bailey, M. et al. International Trade and Social Connectedness. Journal of International Economics 129, 1-34 (2021).

23. Kuchler, T., Russel, D. \& Stroebel, J. The Geographic Spread of COVID-19 Correlates with the Structure of Social Networks as Measured by Facebook. Journal of Urban Economics, 1-13 (2020).

24. Charoenwong, B., Kwan, A. \& Pursiainen, V. Social Connections with COVID19-Affected Areas Increase Compliance with Mobility Restrictions. Science Advances 6, 1-10 (2020).

25. Manski, C. F. Identification of Endogenous Social Effects: The Reflection Problem. Review of Economic Studies 60, 531-542 (1993).

26. Jackson, M. O. Social and Economic Networks. Princeton University Press (2008). 
27. Bramoulle, Y., Djebbari, H. \& Fortin, B. Identification of Peer Effects through Social Networks. Journal of Econometrics 150, 41-55 (2009).

28. Bramoulle, Y., Djebbari, H. \& Fortin, B. Peer Effects in Networks: A Survey. Annual Review of Economics 12, 1-27 (2020).

29. Gibbons, S. \& Overman, H. G. Mostly Pointless Spatial Econometrics? Journal of Regional Science 52, 172-191 (2012).

30. Vega, S. H. \& Elhorst, J. P. The SLX Model. Journal of Regional Science 55, $1-25$ (2015).

31. Kelly, M. Understanding Persistence 2020. https : / / economics . yale . edu/ sites/default/files/understanding_persistence_ada-ns.pdf (2021).

32. Hsiang, S. M. Temperatures and Cyclones Strongly Associated with Economic Production in the Caribbean and Central America. Proceedings of the National Academy of Sciences 107, 15367-15372 (2010).

33. Google. COVID-19 Symptoms Search Trends https://pair-code.github. io/covid19_symptom_dataset/?country=GB (2021).

34. American Psychiatric Association. Diagnostic and Statistical Manual of Mental Disorders: Fifth Edition 2013.

35. World Health Organization. The ICD-10 Classification of Mental and Behavioural Disorders 1990.

36. Facebook. Facebook Social Connectedness Index https://data.humdata.org/ dataset/social-connectedness-index (2021).

37. Wahltinez, O. et al. COVID-19 Open-Data https : / goo.gle/covid-19open-data (2021).

38. European Centre for Disease Prevention and Control. COVID-19 datasets https : //www. ecdc.europa.eu/en/covid-19/data (2021).

39. World Health Organization. WHO Coronavirus Disease (COVID-19) Dashboard https://covid19. who.int/ (2021).

40. Roser, M., Ritchie, H., Ortiz-Ospina, E. \& Hasell, J. Coronavirus Pandemic (COVID-19) https://ourworldindata.org/coronavirus (2021).

41. The New York Times. Coronavirus (Covid-19) Data in the United States https: //github.com/nytimes/covid-19-data (2021).

42. Hale, T. et al. A Global Panel Database of Pandemic Policies (Oxford COVID19 Government Response Tracker) https://www.bsg.ox.ac.uk/research/ research-projects/covid-19-government-response-tracker (2021).

43. US Census Bureau. County-to-County Migration Flows: 2014-2018 ACS 2021. https://www. census.gov/data/tables/2018/demo/geographic-mobility/ county-to-county-migration-2014-2018.html (2021).

44. Ruggles, S. et al. IPUMS USA: Version 11.02021. 
45. US Census Bureau. Cartographic Boundary Files - Shapefile 2018. https:// www . census . gov/geographies/mapping-files/time-series/geo/cartoboundary-file.html (2021).

46. Pearl, J. Causality: Models, Reasoning, and Inference (2009).

47. Montgomery, J. M., Nyhan, B. \& Torres, M. How Conditioning on Posttreatment Variables Can Ruin Your Experiment and What to Do about It. American Journal of Political Science, 1-16 (2018).

48. Schisterman, E. F., Cole, S. R. \& Platt, R. W. Overadjustment Bias and Unnecessary Adjustment in Epidemiologic Studies. Epidemiology 20, 488-495 (2009). 